this is present, treating it, perhaps curing it, or when the condition is grave and untreatable and in keeping with the unbearable anxiety that it generates in the mother, or better the parents, and in conformity with their wishes, terminating the undesired pregnancy at a stage when this is morally and legally acceptable. Disregarding the detection of gross developmental malformations by contrast radiography, ultrasonic scanning or fetoscopy, the favoured procedure for the detection of anomalies leading to serious and intractable handicap is early amniocentesis. The study of the fluid and cells is useful in respect of four main groups of anomalies.

First, study of the amniotic fluid and cells can be useful for the detection of mutants of large effect which are responsible for the severe inborn errors of metabolism, often untreatable and generally incurable and which have a high recurrence risk in certain families. Secondly, there is the detection of $\mathrm{X}$-linked disease which often-but there are exceptions like Lesch-Nyhan's or Hunter's diseaseis less precise because indirect. Thirdly there is the detection of chromosome anomalies of which the most relevant is trisomy-21(mongolism), because of the high prospect of severe subnormality and its frequency and the high risk of its recurrence in a few families. Fourthly, there seems to be now the chance of an early detection of anencephaly and some forms of spina bifida cystica by demonstrating in early pregnancy an excess of fetal $\alpha$-protein in the amniotic fluid.

Prenatal diagnosis of chromosome and sex-linked disorders, and especially mongolism, will be discussed.

\section{Symposium IV}

\section{Encephalitis and meningitis}

Cryptococcal and Other Forms of Mycotic Meningitis W. ST. C. SYMMERS (Department of Pathology, Charing Cross Hospital and Medical School) What we conventionally refer to as fungal meningitis is, of course, meningoencephalitis. It is important to remember this as treatment that seems effective against the meningeal component of the illness may not sterilize lesions within the brain: these may subsequently be the source of reinfection of the meninges.

Fungal meningoencephalitis may develop in the absence of apparent infection elsewhere, or it may be incidental in the course of a generalized haematogenous mycosis. It may occur without predisposing factors, or it may be an 'opportunistic' infection, predisposed to by the resistance-lowering effects of other diseases or of their treatment.
Some fungi have a predilection for the central nervous system-Cryptococcus neoformans, Cladosporium bantianum, the 'opportunist' phycomycetes (species of Rhizopus, Absidia and Mucor) and Nocardia asteroides. Others (for instance, species of Aspergillus and of Candida, the histoplasmas and Coccidioides immitis) have less affinity for the central nervous system and infect it comparatively seldom: when they do so, this may be the presenting or even the only clinical manifestation of the infection, orusually as part of a generalized bloodstream infection-it may be accompanied by little or no evidence of neurological disturbance.

A series of cases, all seen in Britain, is presented, including actinomycosis, nocardiosis, streptomycosis, madurellosis, aspergillosis, penicilliosis, phycomycosis, candidosis, geotrichosis, cryptococcosis, North American blastomycosis, chromomycosis, and sporotrichosis. Some cases are also noted in which the microscopical appearances of the organisms were not familiar and cultures were not obtained: for the moment they must be added to the number of mycoses caused by as yet unidentified fungi.

Among the 'opportunistic' fungal infections of the central nervous system, special attention is due to naso-orbitocerebral phycomycosis complicating sustained acidosis, particularly in diabetes mellitus. The same sequence of nasal, orbital, and meningocerebral infection is occasionally caused by aspergilli, particularly Aspergillus flavus: this species is a cause of infection of the nasal sinuses, particularly in hot, dry climates-its spread to the orbit and the brain is not necessarily related to predisposing factors, in contrast to the phycomycetes.

Double and multiple opportunistic fungal infections of the central nervous system are not infrequent. Any combination of 'opportunist' moulds, yeasts, and actinomycetes may be found, and opportunistic bacterial, viral, and protozoal infections-and even metazoan infestations-may coexist. As examples, two cases recently seen in Britain are presented: a case of meningoencephalitis caused by a free-living amoeba (Naegleria species) superimposed on cryptococcal meningoencephalitis complicating sardoidosis; and a case of anomalous haematogenous infestation by larvae of Strongyloides stercoralis associated with septicaemic candidosis as complications of lymphatic laukaemia under treatment with cytotoxic drugs and corticosteroids.

Meningococcal Infection: Serotypes and Suplhonamide Sensitivity J. D. ABBoTt (Public Health Laboratory, Withington Hospital, Manchester) In recent years sulphonamide resistance of meningococci has become a problem in the USA and elsewhere. Since 